\title{
The Secret of the Copper Box: public experiments and the prospects of living archives of Swedish nuclear waste
}

\author{
Thomas P. Keating and Anna Storm \\ Department of Thematic Studies: Technology and Social Change (TEMA T), \\ Linköping University, 58183 Linköping, Sweden
}

Correspondence: Thomas P. Keating (thomas.keating@liu.se) and Anna Storm (anna.storm@liu.se)

Published: 10 November 2021

\begin{abstract}
This paper approaches the question of nuclear safety in relation to the prospects of living archives, and in particular, it explores two public events where sociocultural aspects of nuclear waste management in Sweden were enacted. Drawing on an ongoing research collaboration with the Swedish Nuclear Fuel and Waste Management Company (SKB), the paper examines (1) a grass-roots play entitled Kopparkistans hemlighet (or The Secret of the Copper Box), and (2) a drawing competition hosted by schools located near a nuclear waste storage facility in the Östhammar municipality, considering how these events help reproduce certain kinds of social and cultural responsibility. Contributing to critical debates in the social sciences and humanities intersecting questions of memory preservation, nuclear waste, and post-nuclear natures, we consider how the forms of responsibility produced through these public experiments inform important, albeit unconventional, modes of nuclear waste management insofar as they suggest how long-lived nuclear waste sites might become living archives without assuming a narrow notion of heritage and human memory preservation for all time and space. To conclude, we evaluate how the legacy of the two events might be incorporated into wider strategies of nuclear waste management.
\end{abstract}

Kurzfassung. Der vorliegende Beitrag befasst sich mit der Frage der nuklearen Sicherheit im Zusammenhang mit den Möglichkeiten von „Living Archives“ und untersucht insbesondere zwei öffentliche Veranstaltungen, bei denen soziokulturelle Aspekte der Entsorgung nuklearer Abfälle in Schweden thematisiert wurden. Auf der Grundlage einer laufenden Forschungskooperation mit der Schwedischen Gesellschaft für Kernbrennstoff- und Abfallmanagement (SKB) untersucht der Beitrag (1) ein Theaterstück mit dem Titel Kopparkistans hemlighet (oder Das Geheimnis der Kupferkiste) und (2) einen Malwettbewerb, der von Schulen in der Nähe eines Atommülllagers in der Gemeinde Östhammar veranstaltet wurde, und geht der Frage nach, wie diese Veranstaltungen dazu beitragen können, bestimmte Arten von sozialer und kultureller Verantwortung zu reproduzieren. Als Beitrag zu den kritischen Debatten in den Sozial- und Geisteswissenschaften, die sich mit Fragen der Bewahrung der Erinnerung, des Atommülls und der postnuklearen Natur auseinandersetzen, betrachten wir, wie die Formen der Verantwortung, die durch diese öffentlichen Experimente entstehen, wichtige, wenn auch unkonventionelle Formen der Atommüllentsorgung aufzeigen, da sie vorschlagen, wie Atommüllendlager zu lebendigen Archiven werden könnten, ohne ein enges Konzept von der Bewahrung des Erbes und der menschlichen Erinnerung für alle Zeiten und Räume vorauszusetzen. Abschließend bewerten wir, wie das Vermächtnis der beiden Veranstaltungen in weiter gefasste Entsorgungsstrategien für nuklearen Abfall integriert werden könnte. 
Financial support. This research has been supported by the Svensk Kärnbränslehantering (grant no. 24992). 